Draft VERSION FEBRUARY 14, 2019

Typeset using IATEX RNAAS style in AASTeX62

\title{
Mid-INFARED LIGHT CURVE AND HIGH-RESOlution RADIO STRUCTURE OF THE CANDIDATE NEUTRINO SOURCE GB6 J1040+0617
}

\author{
Krisztina É. Gabányi, ${ }^{1,2}$ Sándor Frey, ${ }^{2}$ and Attila Moór ${ }^{2}$ \\ ${ }^{1}$ MTA-ELTE Extragalactic Astrophysics Research Group, Pázmány Péter sétány 1/A, H-1117 Budapest, Hungary \\ ${ }^{2}$ Konkoly Observatory, MTA CSFK, Konkoly-Thege Miklós út 15-17, H-1121 Budapest, Hungary
}

Keywords: BL Lacertae objects: individual: GB6 J1040+0617 - infrared: galaxies - radio continuum: galaxies

Recently, Garrappa et al. (2019) published a multi-wavelength study of the neutrino event IceCube-141209A. They found a $\gamma$-ray source spatially coincident and showing optical flare and brightening in $\gamma$-rays that were nearly simultaneous with the neutrino event. This source is associated with the radio-loud active galactic nucleus (AGN) GB6 J1040+0617 (at redshift $z=0.7351 \pm 0.0045$, Maselli et al. 2015). Based upon the multi-wavelength characteristics, Garrappa et al. (2019) conclude that GB6 J1040+0617 is a more probable counterpart of the neutrino event IceCube-141209A than the close-by other radio-loud AGN, 4C+06.41. Similar to the other neutrino source candidate, TXS 0506+056 (Aartsen et al. 2018), GB6 J1040+0617 is a BL Lacertae type object, because its radio jet is seen at small angle to the line of sight.

To search for possible simultaneous mid-infrared variability of GB6 J1040+0617, we analyzed the W1 (3.4 $\mu \mathrm{m})$ and W2 $(4.6 \mu \mathrm{m})$ band data of the Wide-Field Infrared Survey Explorer (WISE, Wright et al. 2010; Mainzer et al. 2014). We refer to Gabányi et al. (2018b) for the details of the observations and data analysis technique. Most of the WISE measurements of GB6 J1040+0617 had a signal-to-noise ratio (SNR) larger than 10. We discarded from the analysis three points in W2 band having SNR $<2$. The resulting light curves contain 109 and 106 measurements in bands W1 and W2, respectively. The individual mission phases contain $11-20$ data points and last for $1-4$ days.

After converting the magnitudes to Jy, we calculated the weighted averages, standard deviations and reduced $\chi^{2}$ values for each mission phase separately in both bands. The resulting light curves are shown in Fig. 1. GB6 J1040+0617 is variable in both mid-infrared bands on year-long time scale. Notably, it was brightest, increasing its flux density by almost $90 \%$, in the mission phase preceding the neutrino event IceCube-141209A by $4.5 \mathrm{~d}$. This brightening in midinfrared is thus coincident with the optical flare and $\gamma$-ray brightening of the source (Garrappa et al. 2019). The other neutrino source candidate, TXS 0506+056 showed similar behavior, brightening in the W1 and W2 bands preceding its neutrino event IceCube-170922A (Gabányi et al. 2018b).

We considered the source showing variability in a given mission phase if the reduced $\chi^{2}$ value was larger than 3 (Gabányi et al. 2018a). The reduced $\chi^{2}$ values exceed 3 for most mission phases in both bands, thus indicating variability on time scales less than a day in GB6 J1040+0617. The short variability time scale implies a small emitting region indicating that the jet is likely to be responsible for the variability (Jiang et al. 2012; Yang et al. 2018; Gabányi et al. 2018a). There is no indication of short time scale variability (reduced $\chi^{2}<3$ ) in either of the bands in the mission phases directly preceding and following the neutrino event IceCube-141209A.

Milliarcsecond-scale radio images of GB6 J1040+0617 were obtained with the Very Long Baseline Array (VLBA) more than 2 years before the neutrino event. The source was observed at $1.4 \mathrm{GHz}$ on 2012 February 24 within the mJy Imaging VLBA Exploration (mJIVE, Deller \& Middelberg 2014) program, and at $8.4 \mathrm{GHz}$ on 2012 March 13 within the 2MASS VLBA observing program (Condon et al. 2011) ${ }^{1}$. The source shows a core-jet structure at both frequencies. We used circular Gaussian brightness distribution model components to fit the visibility data in

Corresponding author: Krisztina É. Gabányi

krisztina.g@gmail.com

1 The calibrated datasets can be obtained from http://safe.nrao.edu/vlba/mjivs/home.html and http://astrogeo.org, respectively. 
Difmap (Shepherd et al. 1994). For both datasets, a circular core and an elliptical jet feature adequately describe the data. The core component has flux densities of $S_{1.4 \mathrm{GHz}}=25.7 \pm 3.0 \mathrm{mJy}$ and $S_{8.4 \mathrm{GHz}}=30.8 \pm 0.5 \mathrm{mJy}$ at 1.4 , and $8.4 \mathrm{GHz}$ respectively, implying a slightly inverted radio spectrum. The brightness temperature of the $8-\mathrm{GHz}$ core is $T_{\mathrm{B}}=1.22 \times 10^{9}(1+z) S_{8.4 \mathrm{GHz}} \theta^{-2} \nu^{-2} \approx 2.3 \times 10^{10} \mathrm{~K}$, where $\theta=0.20 \pm 0.03$ mas is the full width at half maximum size of the fitted component, and $\nu$ is the observing frequency in GHz. The brightness temperature is somewhat below the equipartition value, $\sim 5 \times 10^{10} \mathrm{~K}$ (Readhead 1994). While $\gamma$-ray bright blazars tend to have brightness temperatures exceeding the equipartition limit, there are sources with values similar to that of GB6 J1040+0617 (Böck et al. 2016 , and references therein).

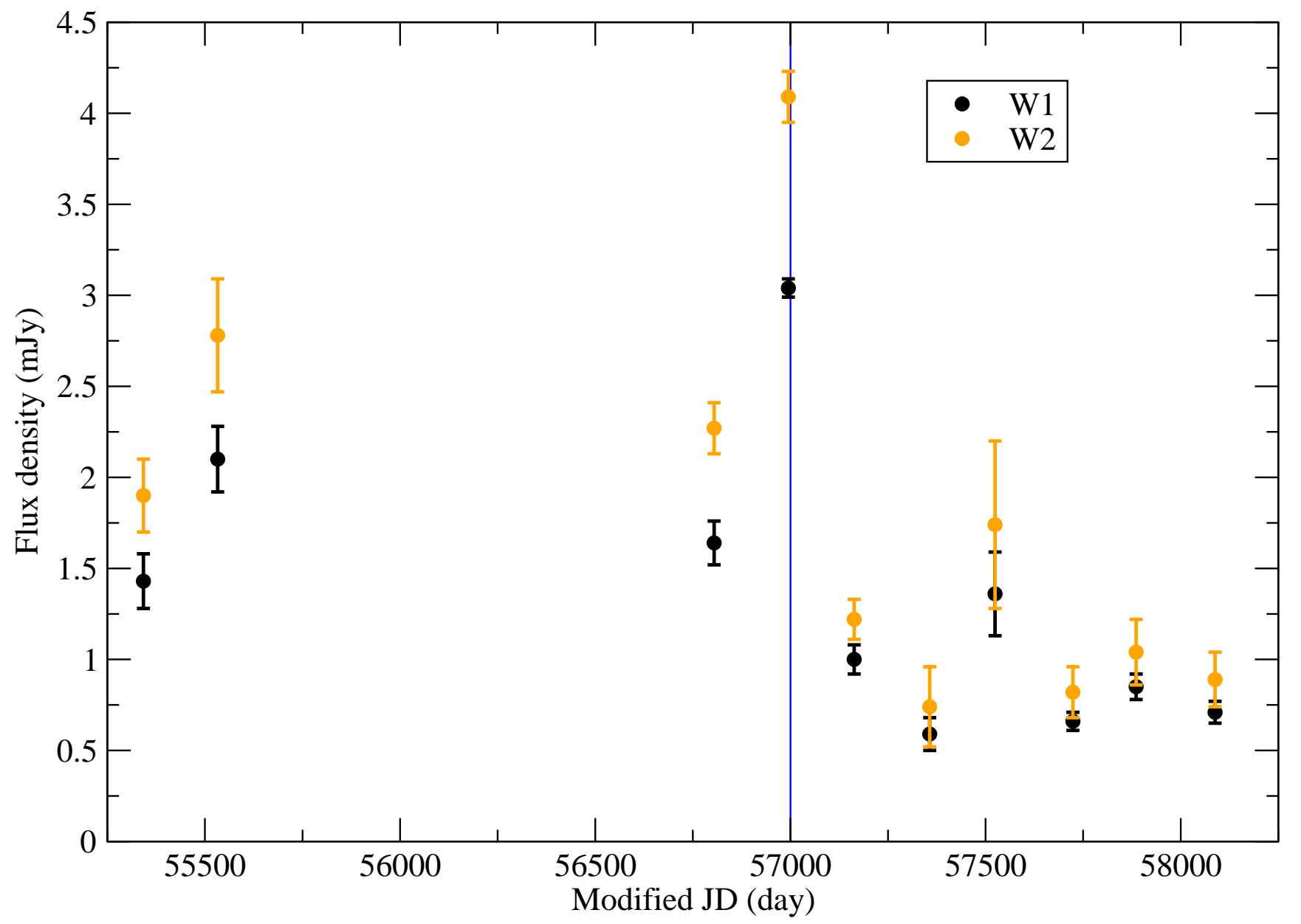

Figure 1. WISE light curves of GB6 J1040+0617, black and orange symbols are for bands W1 and W2, respectively. Points are averages of each mission phase, the error bars represent the variability within the given phases. Blue line marks the time of the neutrino event IceCube-141209A.

KÉG acknowledges the János Bolyai Research Scholarship of the Hungarian Academy of Sciences. This work was supported by the NKFIH-OTKA NN110333 grant. This publication makes use of data products from the Wide-field Infrared Survey Explorer, which is a joint project of the University of California, Los Angeles, and the Jet Propulsion Laboratory/California Institute of Technology, funded by the National Aeronautics and Space Administration. We used VLBA data (projects BC201AP, BD161AB) provided by the National Radio Astronomy Observatory that is a facility of the National Science Foundation operated under cooperative agreement by Associated Universities, Inc. 


\section{REFERENCES}

Aartsen, M. G., Ackermann, M., Adams, J., et al. 2018, Science 361 , eaat 1378

Böck, M., Kadler, M., Müller, C., et al. 2016, A\&A, 590, A40

Condon, J., Darling, J., Kovalev, Y. Y., et al. 2011, NARIT Conference Series, 1, 393

Deller, A. T., \& Middelberg, E. 2014, AJ, 147, 14

Gabányi, K. É., Moór, A., \& Frey, S. 2018a, Proceedings of Science, PoS(NLS1-2018)042

Gabányi, K. É., Moór, A., \& Frey, S. 2018b, RNAAS, 2, 130
Garrappa, S., Buson, S., Franckowiak, A., et al. 2019, arXiv e-prints, arXiv:1901.10806

Jiang, N., Zhou, H.-Y., Ho, L. C., et al. 2012, ApJ, 759, L31

Mainzer, A., Bauer, J., Cutri, R. M., et al. 2014, AJ, 792, 30

Maselli, A., Massaro, F., D'Abrusco, R., et al. 2015, Ap\&SS, 357, 141

Readhead, A. C. S. 1994, ApJ, 426, 51

Shepherd, M. C., Pearson, T. J., \& Taylor, G. B. 1994, BAAS 26, 987

Wright, E. L., Eisenhardt, P. R. M., Mainzer, A. K., et al. 2010, ApJ, 140, 1868

Yang, H., Yuan, W., Yao, S., et al. 2018, MNRAS, 477, 5127 